\title{
Die Wühlmaus wühlt weiter...
}

\section{Mit Anawülasis Geschäftsgänge digitalisieren, landesbibliothekarische Aufgaben erfüllen, Quellen lieferantenunabhängig auswerten und Medien erwerben}

Im Oktober 2017 hat die Württembergische Landesbibliothek (WLB) das Tool "Anawülasis" als das neue Auswertesystem für Titeldaten in Betrieb genommen. Das als Eigenentwicklung entstandene Programm ermöglicht den Fachreferenten und der Medienbearbeitung die Auswahl bzw. Erwerbung von Medien und erfüllt gleichzeitig landesbibliothekarische Aufgaben. Basis sind die Reihen der Deutschen Nationalbibliografie und Buchhandelsdaten.

In der WLB wird Anawülasis für vier Arbeitsbereiche verwendet: Es unterstützt die Abteilung Medienbearbeitung Pflicht (MB Pflicht) bei der Reklamation von Pflichttiteln, die Abteilung Landesbibliographie bei der Auswahl landeskundlich relevanter Titel, die Fachreferenten und die Medienbearbeitung Kauf (MB Kauf) bei Auswahl bzw.
Erwerbung deutschsprachiger Literatur. Das Programm ist dabei offen konzipiert und ermöglicht die Anbindung weiterer Titelquellen. Bereits umgesetzt ist die Integration der englischsprachigen Literatur der Buchhandlung Dreier.

Abbildung 1 bietet einen Überblick über den Workflow mit Anawülasis: Die Aufbereitung und Verteilung der Titeldaten erfolgt direkt nach dem Abholen der Titeldaten von den Lieferanten. Anschließend steht ein Titel entweder der MB Pflicht oder gleichzeitig der Landesbibliographie und den Fachreferenten zur Verfügung. Anawülasis ist dabei ein "Titellieferant" - es liefert ausschließlich "Hinweise" auf Titel. Die weitere Bearbeitung erfolgt anschließend in anderen Systemen: die Katalogisierung in der Verbund-Datenbank (WinIBW), die Bestellung bzw. Reklamation im Lokalsystem (aDIS/BMS) und die

\section{ANAWÜLASIS}

WLB-Auswertetool für DNB-Reihen

Übersicht Geschäftsgang WLB

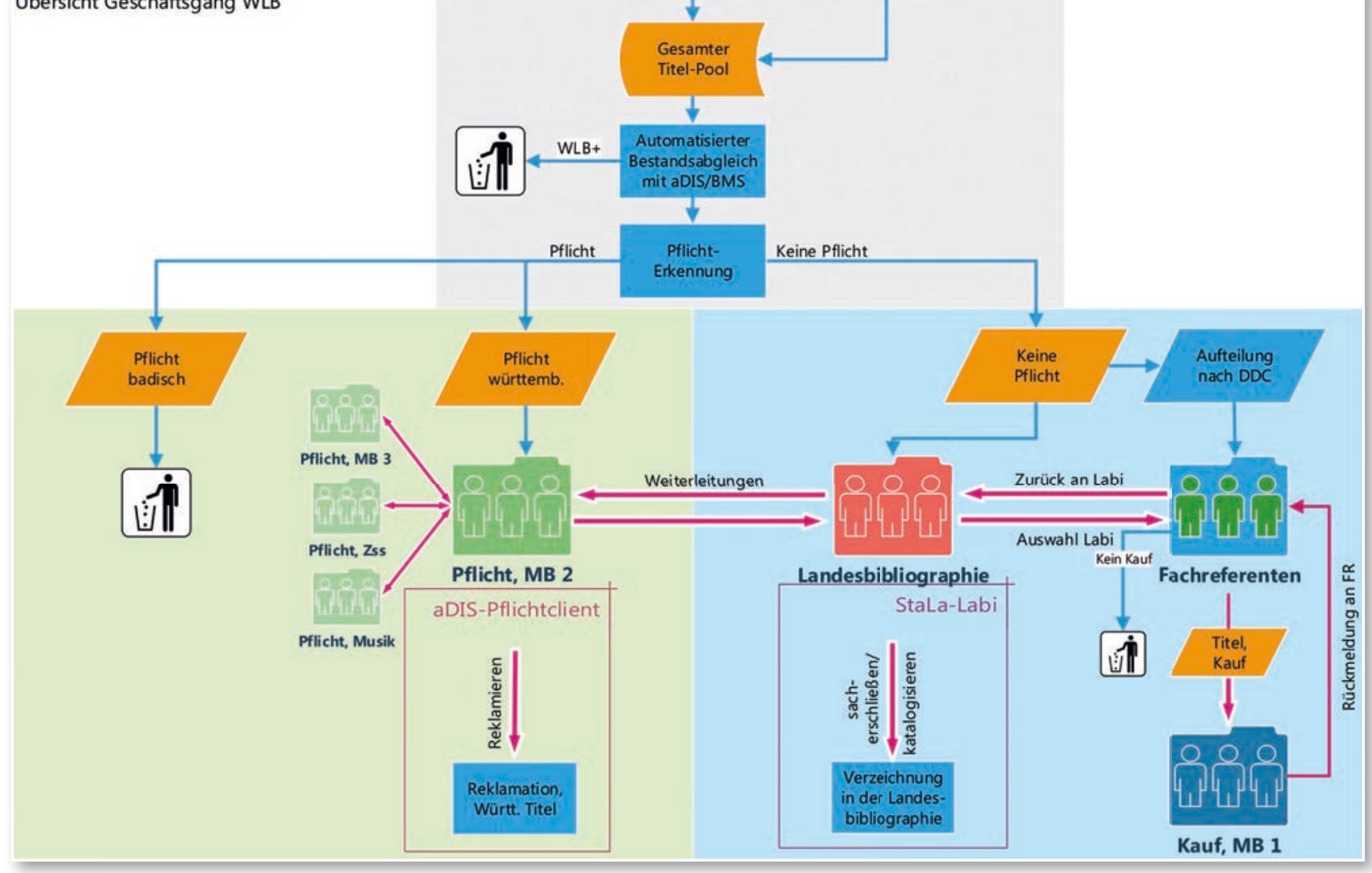

Abb. 1: Workflow Anawülasis inkl. Anbindung an aDIS/BMS und StaLa-Client. Zwischen allen Modulen gibt es die Möglichkeit der automatischen und/oder manuellen Weiterleitung von Titeln. 
Aufnahme in die Landesbibliographie mit dem StaLa-Client des Statistischen Landesamtes (StaLa). Den Mitarbeitern steht zur Bearbeitung eine Weboberfläche zur Verfügung, wie sie - in Ausschnitten - in Abb. 2 und 3 zu sehen sind. Die Weboberflächen sind in allen Modulen grundsätzlich gleich aufgebaut; sie unterscheiden sich lediglich in den verfügbaren Titellisten und den jeweils möglichen Aktionen. Grundsätzlich gilt, dass jeder Titel bearbeitet, d.h. irgendeine Aktion durchgeführt werden muss. Dazu stehen modulabhängig pro Titel Einzelaktionen wie „Reklamieren“, „Merkliste”, "Weiterleiten", "Kaufen“ oder „Nicht Kaufen“ zur Verfügung. Zudem gibt es sogenannte Massenfunktionen zum Bearbeiten aller Titel auf einer Bildschirmseite, z. B. "Nicht kaufen" oder "Alle landeskundlich relevant". Für weitere Informationen sei auf den zuletzt erschienenen Artikel ${ }^{1}$ verwiesen. Im vorliegenden Bericht werden die internen Geschäftsgänge mit Anawülasis für die vier Bereiche Pflicht, Landesbibliographie, Fachreferate und Kauf näher vorgestellt. Außerdem wird über die wichtigsten Neuerungen der letzten Monate berichtet.

\section{Weiterentwicklungen der letzten Versionen}

Seit dem Start des Produktivbetriebs von Anawülasis im Oktober 2017 hat sich einiges getan. Neben zahlreichen kleineren und größeren Neuerungen hat das Projektteam² einigen Aufwand für Optimierungen des Systems investiert. Die meisten Nutzer-Anforderungen konnten inzwischen umgesetzt werden.

\section{Allgemeine Neuerungen}

In der Endnutzeroberfläche wurden einige Verbesserungen zur Usability durchgeführt.

- Dialogfenster und Buttons wurden angeglichen, z.B. bei den Aktionen zum Löschen von Titeln (Abb. 2 oben).

- Neben den Listen, in denen es zu bearbeitende Titel gibt, erscheinen rote Ausrufezeichen (Abb. 3).

- In den persönlichen Einstellungen kann jeder Mitarbeiter wählen, ob alle Kommentare oder nur der neueste Kommentar oder gar kein Kommentar angezeigt werden soll.

- Per Klick auf ein Fernglas-Symbol in der Titelanzeige kann eine Suche nach weiteren Ausgaben im Südwestdeutschen Bibliotheksverbund (SWB) durchgeführt werden. ${ }^{3}$

Der Bestandsabgleich wurde erneut erweitert: Be- rücksichtigt werden nun auch parallele Ausgaben, d. h. es wird angezeigt, wenn der Titel bereits als E-Book vorhanden ist.

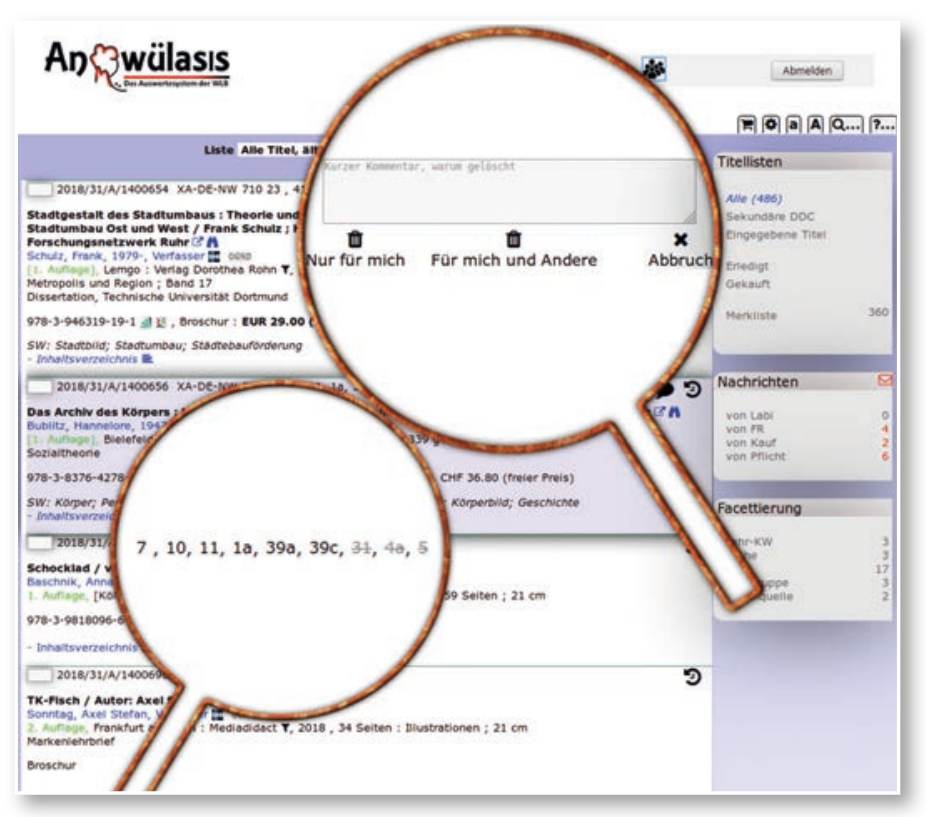

Abb. 2: Benutzeroberfläche im Modul Fachreferenten mit optimierten Funktionen und Anzeigen. Oben: Neuer Dialog zum Löschen von Titeln; unten: Fachgruppen, bei denen der Titel bereits gelöscht ist, sind durchgestrichen

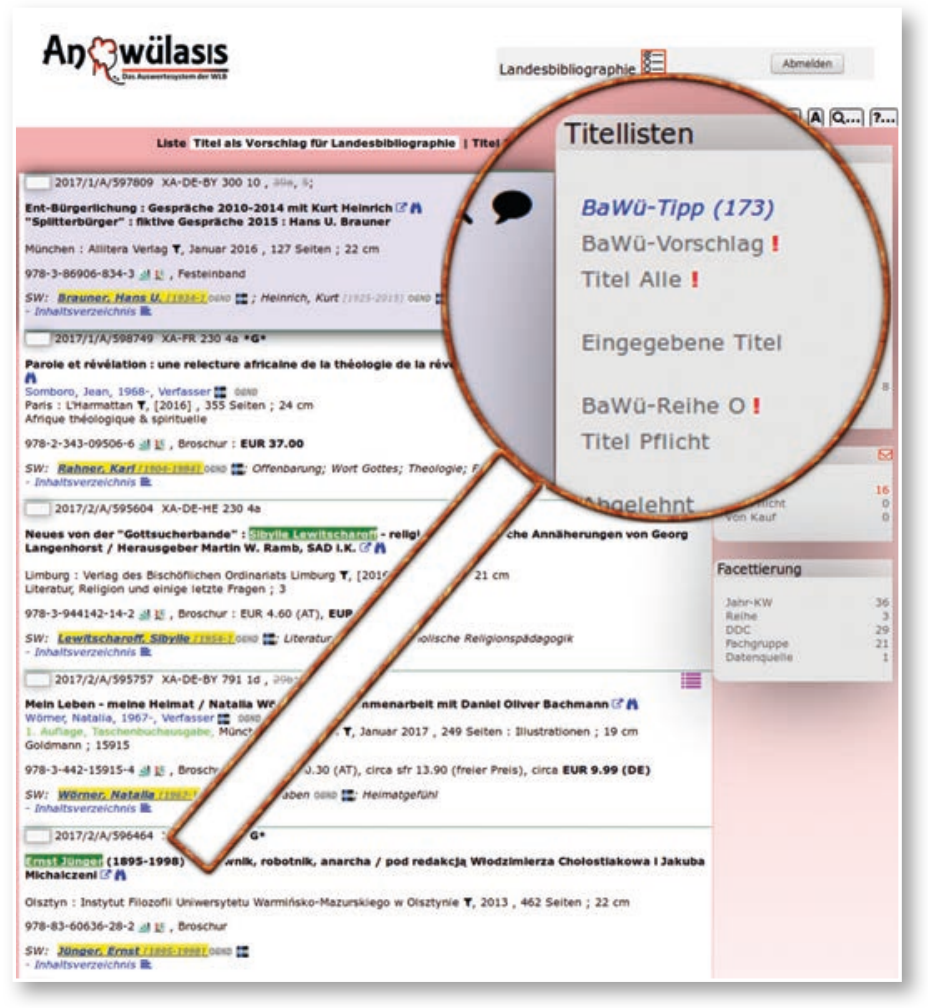

Abb. 3: Nutzeroberfläche im Modul Landesbibliographie. Rotes Ausrufezeichen bei noch zu bearbeitenden Listen

1) Oberfell, Jörg; Thalhofer, Anja: Mit der Wühlmaus "Anawülasis" unterwegs : Das neue Auswertesystem der WLB für Titeldaten. In: WLBforum. - 2018, 1, Seite 7-19.

2) Zum Projektteam gehören Dr. Anja Thalhofer (Arbeitsstelle Landesbibliographie) als Projektleiterin, Jörg Oberfell (IT-Abteilung) und Heinrich Schweitzer (Webentwickler). Unterstützt wird es durch das Knowhow der beteiligten Abteilungen MB Pflicht, Landesbibliographie, Fachreferenten und MB Kauf.

3) Dabei werden der Nachname des Autors sowie der Haupttitel an die Suche übergeben. 


\section{Neuerungen für das Modul Pflicht}

Die Abteilung MB Pflicht ist die zentrale Stelle für die Bearbeitung von Pflichttiteln. Nicht-Buch- und Sondermaterialien sowie Zeitschriften werden von dort an die zuständigen Abteilungen bzw. in die entsprechenden Module weitergeleitet. Neu hinzugekommen ist die Möglichkeit, Titel auch an die Musiksammlung weiterzuleiten, ohne den Status „Pflicht" zu verlassen. ${ }^{4}$ Mit einer weiteren Massenfunktion kann der Bearbeiter festlegen, dass alle Titel mit identischer Schreibweise von Erscheinungsort und Verlag als "keine Pflicht" gekennzeichnet werden sollen.

\section{Neuerungen für die Module Fachreferate und Landesbibliographie}

Bei Titeln, die mehrere Fachreferenten betreffen, werden in der Statuszeile alle beteiligten Fachgruppen angezeigt. Fachgruppen, in denen der Titel bereits gelöscht wurde, werden durchgestrichen dargestellt. Damit werden Weiterleitungen vermieden (Abb. 2 unten).

Ist der Titel zusätzlich landeskundlich relevant, sollte der letzte Fachreferent einen kurzen Kommentar abgeben, warum der Titel nicht gekauft wird. Das erleichtert die Bearbeitung in der Landesbibliographie und vermeidet Rückfragen. Die Funktion zum Massenlöschen wurde entsprechend angepasst: Beim letzten Fachreferenten werden landeskundliche Titel nicht automatisch mitgelöscht.

\section{Neuerungen für das Modul Kauf}

Die Mitarbeiteroberflächen für die MB Kauf wurden überarbeitet. Neben der ursprünglichen "Standardansicht" mit allen Titel-Informationen gibt es nun wahlweise eine Ansicht, bei der nur noch die für den Kauf wesentlichen Titelbestandteile angezeigt werden (Abb. 10).

Im Kaufmodul können Mitarbeiter ein oder mehrere Titel sperren, bevor sie bearbeitet werden. Damit andere Mitarbeiter schneller sehen, ob ein Titel gesperrt ist, erscheint das entsprechende Icon (rotes Schloss) jetzt sofort bei allen gesperrten Titeln. Die Synchronisation erfolgt in Sekundenbruchteilen über den eingebauten Socketserver.

\section{Integration von Fremddaten}

Die Übernahme von Fremddaten der Buchhandlung Dreier als Pilotlieferant wurde durch einzelne

4) Das Modul Musik ist einsatzbereit und für die Meldungen von Noten oder Liederbüchern vorgesehen

5) Basis ist die Zuordnung von Gemeinden und Gemeindeteilen zu den Regierungsbezirken Stuttgart und Tübingen als württembergische Pflicht sowie Karlsruhe und Freiburg als badische Pflicht.
Fachreferenten und die MB Kauf getestet. Nach einigen Anpassungen an Datenfeldern und Bestandsabgleich wurde der Produktivbetrieb im Juli 2018 gestartet und das bisherige Verfahren mit gedruckten Zetteln eingestellt. Die Digitalisierung führt nicht nur zu einer Einsparung riesiger $\mathrm{Pa}$ pierberge, sondern vor allem zu einer deutlichen Arbeitsentlastung in der WLB und in der Buchhandlung Dreier.

Der Import der Dreier-Titeldaten ist ein Prototyp für die Aufnahme weiterer Titeldaten nach Anawülasis. Liegen die Titel im MARC21-Format vor, sind praktisch keine Anpassungen nötig, um die Daten mit Anawülasis zu verarbeiten. Ansonsten ist ein Mapping in ein vereinfachtes Importformat nötig, das nur 10 Felder (Titel, Autor, Verlag usw.) enthält.

Maschinelle Erkennung landeskundlicher Titel Nachdem die Erkennung von landeskundlichen Titeln über den Baden-Württemberg-Index erfolgreich für baden-württembergische Personen und Orte implementiert wurde, konnte das Verfahren für einen Teil der Körperschaften erweitert werden. Es handelt sich dabei um Einträge aus der Körperschaftsdatei der Landesbibliographie mit GND-ID. Körperschaften ohne GND-ID sollen maschinell - durch einen Abgleich mit der GND - mit Normdaten ergänzt werden, um diese ebenfalls für Anawülasis nutzen zu können.

\section{Die Arbeit mit dem Modul Pflicht Was liefert Anawülasis?}

Anawülasis erkennt Titel, die in Baden-Württemberg erschienen sind. In der MB Pflicht dient Anawülasis zur Vorauswahl von württembergischen und badischen Pflichttiteln auf Basis der Reihen A und $B$ der DNB. Die Anwendung ist damit Lieferant württembergischer Pflichttitel, die durch die WLB bei den Verlagen reklamiert werden müssen. Anawülasis differenziert zwei Arbeitsbereiche und liefert dafür mehrere Titellisten (Abb. 4): Titel, die nach der automatischen Pflichterkennung nicht eindeutig als Pflicht erkannt worden sind, werden in den beiden Listen unter "Pflicht prüfen" ausgegeben. Sie enthalten Titel, die vermutlich württembergisch bzw. badisch sind. ${ }^{5}$ Die Listen unter "Reklamieren" beinhalten Titel, die direkt ins Reklamationsverfahren übernommen werden. 
Dabei handelt es sich um Medien, die in den Regierungsbezirken Stuttgart oder Tübingen erschienen sind.

\section{Titellisten}

\section{Pflicht prüfen \\ Württembergisch ? (11) \\ Badisch ?!}

\section{Reklamieren}

\section{Älteste zuerst !}

nach Verlag

Bearbeitet

\section{Merkliste}

Abb. 4: Zu bearbeitende Titellisten im Modul Pflicht

\section{Wie wird mit Anawülasis gearbeitet?}

1. Damit Nicht-Pflicht-Titel möglichst schnell für andere Module bereitgestellt werden können, werden Titel der Listen „Pflicht prüfen“ wöchentlich bearbeitet. Es handelt sich dabei um Titel, die nicht eindeutig zugeordnet werden konnten, weil beispielsweise eine Namensvariante des Verlages nicht in der aDIS-Lieferantendatei gespeichert war oder weil bei häufigen gleichnamigen Ortsnamen eine intellektuelle Entscheidung vorgesehen ist. Jeder Titel dieser Liste kann dann entweder als Nicht-Pflicht (KP, Keine Pflicht), als württembergische oder badische Pflicht eingestuft werden (Abb. 5). Über eine Massenfunktion können außerdem alle Titel mit derselben Schreibweise von Erscheinungsort und Verlag einem der Pflichtbereiche (entweder badisch: BA oder württembergisch: WA oder keine Pflicht: KPA) zugeordnet werden. Damit wird dann eine datenbankinterne, lernende Tabelle gefüllt, über die Titel mit genau dieser Verlags- und Ortsbezeichnung beim nächsten Titelimport automatisiert erkannt und der entsprechenden eindeutigen Liste zugeordnet werden können. 2. Zu reklamierende Titel werden nach einer Verweilfrist von etwa 9 Monaten aus der Liste "Reklamieren - Älteste zuerst" aufgerufen (Abb. 4). Da mit dem Initialimport in Anawülasis Titel ab der Wochenlieferung 01/2016 ins System eingespielt wurden, können auch noch ältere Titel des Jahr-
Abb. 5: Auswahlmenü zur Einstufung von Titeln, die über die automatisierte Pflichterkennung nicht eindeutig erkannt wurden

gangs 2017 rückwirkend mit dem elektronischen Anawülasis-Geschäftsgang bearbeitet werden. Dazu werden alle Titel auch auf ihre Medienart geprüft und beispielsweise Zeitschriften an die Zeitschriftenstelle und Medienkombinationen oder CD-ROMs an die Medienbearbeitung Sondermaterialien weitergeleitet, die jeweils über ein Modul in Anawülasis angegliedert sind.

In Anawülasis wird ein Pflichttitel geprüft, und wenn er zu reklamieren ist mit dem Icon „blaues Megaphon" (Abb. 6) markiert. Das anschließende Mahnverfahren wird konventionell unter Zuhilfenahme von Lieferantendaten aus dem Lokalsystem aDIS/BMS abgewickelt: Der Titel wird zunächst im SWB auf Bestand abgeprüft. Ist er nicht vorhanden, wird ein Reklamationsbrief erstellt oder eine E-Mail erzeugt. Die Titeldaten werden dabei durch Kopieren aus Anawülasis in den Reklamationsbrief übernommen.

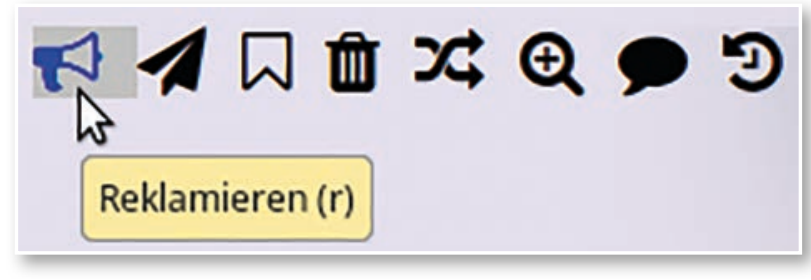

Abb. 6: Aktionsbuttons für die Abteilung Pflicht

Am Ende des Geschäftsgangs wird der reklamierte Titel in Anawülasis auf den Status "reklamiert" gesetzt (erneuter Klick auf das Icon „blaues Megaphon", Abb. 6) und damit aus dem Modul Pflicht entfernt.

Titel, die nicht reklamiert werden können oder müssen, können sofort (über das Icon Papierkorb) aus der Liste entfernt werden. Titel, die sich als Nicht-Pflicht herausgestellt haben, werden mit dem „Doppelpfeil“ als Nicht-Pflicht neu eingestuft. Diese werden dann automatisch an die Module Fachreferenten und Landesbibliographie weitergeleitet. 
3. Weitergeleitete Titel aus anderen Modulen werden der Pflicht im Menü-Bereich "Nachrichten" angezeigt (Abb. 7). Das können Rückmeldungen zu Titeln aus den anderen Pflichtbereichen Zeitschriften, elektronische und audiovisuelle Medien sein oder Titel, die von der Abteilung Landesbibliographie und den Fachreferenten sowie der MB Kauf zur Prüfung an die Pflicht weitergeleitet werden.

\begin{tabular}{|l} 
Nachrichten \\
von Labi
\end{tabular}

Abb. 7: Nachrichten-Bereich in Anawülasis. Neue Weiterleitungen werden mit einem Icon "Post" und in roter Schrift hervorgehoben.

\section{Welche Vorteile bietet Anawülasis der MB Pflicht?}

Mit dem alten Geschäftsgang unter Verwendung der PDF-Version der Deutschen Nationalbibliografie stand die MB Pflicht an erster Stelle des DNBAuswertung-Workflows und musste alle 2.000 bis 4.000 Titel einer Wochenlieferung intellektuell und schnellstmöglich auf württembergische und badische Pflicht prüfen. Mit Einführung des "Anawülasis-Geschäftsgangs" erfolgt diese Pflichterkennung ${ }^{6}$ nun weitestgehend automatisiert und läuft fast ausnahmslos korrekt. Pro Woche bleiben lediglich 5 bis 20 Titel übrig, die geprüft werden müssen, weil sie aufgrund einer abweichenden Schreibweise nicht eindeutig erkannt wurden oder weil ein neuer Lieferant angezeigt ist. Durch die "lernende" Tabelle für verschiedene Bezeichnungen im Hintergrund ist auch weiterhin mit einer reduzierten Zahl an zu prüfenden Titeln zu rechnen. Verbesserungspotential gibt es noch bei der Unterscheidung von Titeln von inaktiven Lieferanten, die beispielsweise aus BadenWürttemberg weggezogen sind oder bei PflichtVerlagen, bei denen die WLB auf die Entgegennahme der Verlagsproduktion oder eines Teils (z.B. auf bestimmte Fachgebiete) verzichtet. Hier wäre die Lösung, schon in der Lieferantendatei im Lokalsystem aDIS allen betroffenen Verlagen einen entsprechenden Status zu vergeben. Dies ist für die Bearbeitung der Titel durch die Abteilung Landesbibliographie und die Fachreferenten sehr wichtig, damit keine Verzichts-Titel in die Erwerbung gelangen.

Ein weiterer Vorteil von Anawülasis ist die automatisierte Entfernung von Titeln auf der Reklamationsliste durch den regelmäßigen Bestandsabgleich, der zweimal in der Woche auf den sich laufend ändernden Gesamtbestand beider Systeme (Anawülasis und aDIS/BMS) durchgeführt wird. Hiervon profitiert in besonderem Maße die MB Pflicht. Da sie die fehlenden Titel nicht sofort reklamiert, verschwinden die inzwischen gelieferten und erfassten Titel nach und nach aus Anawülasis sodass die Abteilung lediglich die verbleibenden Titel bearbeiten muss. Die Titelliste ist somit stets eine aktuelle Bearbeitungsliste.

Alle Vorteile münden in einer erheblichen Zeit- und Personalersparnis bei der Bearbeitung von Reklamationstiteln. Der Geschäftsgang ist deutlich schneller. Die Zeiteinsparung wirkt sich auf alle anschließenden Abteilungen positiv aus, waren sie doch von den Auswahl-Arbeiten badischer und württembergischer Pflichttitel fachlich und zeitlich abhängig. Landesbibliographie und Fachreferenten konnten ihre Auswertung erst anschließend durchführen.
Eine große Hilfe ist für uns, dass sowohl die pflichtrelevanten Titel zum großen Teil maschinell selektiert werden als auch die Vorakzession bereits durch Anawülasis erledigt wird.

Sehr positiv war für uns außerdem, dass wir von Anfang an in die Entwicklung von Anawülasis miteinbezogen wurden und unsere Wünsche nach Anpassungen im System sehr offen aufgegriffen und entsprechende Tools schnell eingefügt wurden. So wurde für uns z. B. die Möglichkeit eingebaut, bestimmte Titel innerhalb von Anawülasis zur Prüfung an andere Arbeitsbereiche zu schicken und gegebenenfalls von dort auch wieder zurückzubekommen. 


\section{Die Arbeit mit dem Modul Landes- bibliographie Was liefert Anawülasis?}

Anawülasis listet Titel, die landeskundlich relevant sind bzw. sein könnten. Diese werden dann für eine Verzeichnung und Erschließung in der Landesbibliographie Baden-Württemberg geprüft. Im Modul Landesbibliographie werden drei verschiedene Titellisten ausgegeben, die neben den DNB-Reihen A und B zusätzlich Hochschulschriften aus Reihe $\mathrm{H}$ berücksichtigen (Abb. 3):

- eine mit eindeutig landeskundlich relevant erkannten Titeln (BaWü-Tipp),

- eine mit einem vermuteten Landesbezug (BaWü-Vorschlag) und

- eine mit allen übrigen Titeln (Titel alle). Elektronische Publikationen aus der DNB-Reihe 0 mit Landesbezug stehen in einer separaten Liste zur Verfügung (BaWü-Reihe O).

\section{Wie wird mit Anawülasis gearbeitet?}

1. Mit Lieferung der wöchentlichen DNB-Titel werden sofort und als Erstes Titel bearbeitet, die über den Baden-Württemberg-Index als eindeutig relevant in der Liste „BaWü-Tipp“ aufgeführt werden. Dies erfolgt durch eine Erkennung von GND-Nummern im Sacherschließungsbereich der Titeldaten für Personen, Geografika und Körperschaften. Anhand der Aufnahme-Kriterien der Landesbibliographie können einzelne Titel über das Icon "Landeswappen" direkt ausgewählt werden und gelangen automatisiert per One-Klick an den oder die zuständigen Fachreferenten. ${ }^{7}$ Die Auswahl bewirkt außerdem eine Statusänderung des Titels in "relevant für Labi”, die jedem nachfolgenden Bearbeiter angezeigt wird. ${ }^{8}$ Bei umfangreichen Titellisten besteht die Möglichkeit mit Massenfunktionen zu arbeiten: Beispielsweise können Titel, die nicht in der Landesbibliographie verzeichnet werden, zunächst per Icon „Papierkorb" einzeln aus der Liste entfernt werden. Die verbleibenden Titel können anschließend über die Massenfunktion „alle Titel relevant“ an die Fachreferenten geschickt und dadurch aus dem Modul Landesbibliographie entfernt werden.

Die gleiche Bearbeitungsweise erfolgt für die Titelliste „BaWü-Vorschlag”. Diese muss genauer geprüft werden, da hier Titel herausgefiltert werden, die im Titel einen Begriff aus dem Baden-Württemberg-Index als Klartext enthalten.
So werden beispielsweise mit dem Ortsschlagwort für die Gemeinde „Kuchen“ im Landkreis Göppingen auch Titel wie "Torten und Kuchen" markiert. Dennoch bietet die Markierung einen enormen Mehrwert, denn die „falsch“ erkannten Titel sind schneller aussortiert, als alle landeskundlich relevanten Titel zu finden. Alle anderen Titel werden derzeit noch gesichtet, weil die direkte Verlinkung von Inhaltsverzeichnissen insbesondere bei Sammelwerken die Möglichkeit bietet, bereits an dieser Stelle Werke mit inhaltlich relevanten Beiträgen zu finden.

2. Rückmeldungen von Titeln aus anderen Modulen werden im Menü-Bereich „Nachrichten” angezeigt. Dabei handelt es sich zum Beispiel um Titel, für die eine Erwerbung vom Fachreferenten abgelehnt oder im Modul Kauf von der MB Kauf gelöscht wurden, da dieser beispielsweise vergriffen ist. In beiden Fällen werden die betreffenden Titel automatisiert an die Landesbibliographie zurückgeleitet. Das Kommentarfeld bietet in jedem Modul bei jedem Titel die Möglichkeit, den Grund für eine Zurück- oder Weiterleitung anzugeben und erleichtert der empfangenen Abteilung die Weiterarbeit.

3. Neu ist, dass mit Anawülasis auch abteilungsinterne Geschäftsgänge von der Papier-Bearbeitung auf eine elektronische Bearbeitung umgestellt werden konnten. Die erste Änderung betrifft Titel, die nicht erworben werden (können). Diese werden dafür auf eine (Merk-)Liste gesetzt, die Sacherschließung der Landesbibliographie wird in das Kommentarfeld eingetragen und von dort in die Landesbibliographie-Datenbank übernommen. ${ }^{9}$ Daneben können ebenso persönliche Merklisten eingerichtet werden.

\section{Welche Vorteile bietet Anawülasis der Landes- bibliographie?}

Mit dem alten Geschäftsgang (PDF-Version) stand die Abteilung Landesbibliographie an zweiter Stelle des DNB-Auswertung-Workflows und war zeitlich abhängig von den Arbeiten der MB Pflicht. Sämtliche der 2.000 bis 4.000 Titel einer Wochenlieferung der DNB wurden intellektuell auf

7) In der WLB reicht die Abteilung Landesbibliographie Titel mit Landesbezug, die in der Datenbank Landesbibliographie Baden-Württemberg verzeichnet werden, als Kaufvorschlag an die zuständigen Fachreferenten weiter.

8) Im Modul Kauf wird diese Statusmeldung in das Bestellkatalogisat als "betrifft BaWü" übernommen. Mit Eintreffen des Werkes in der Württembergischen Landesbibliothek wird dann zusätzlich der Laufzettel für den Geschäftsgang "Landesbibliographie" eingelegt.

9) Die Verzeichnung erfolgt dann ohne Bestand und ohne Signatur-Angabe der Württembergischen Landesbibliothek. 
Baden-Württembergica zur Verzeichnung in der Landesbibliographie geprüft. Der „Anawülasis-Geschäftsgang" unterstützt die Auswahl von BadenWürttemberg-relevanten Titeln nicht nur, sondern übernimmt die (Vor-)Auswahl weitestgehend automatisiert. Ergebnis sind getrennte Listen für eindeutig relevante Titel bzw. Vorschläge, die mit erheblicher Zeitersparnis geprüft und automatisiert als Erwerbungsvorschlag an den/die Fachreferenten geschickt werden. Bis zur Bestellung eines Mediums können damit weniger als 24 Stunden vergehen, wenn auch der Fachreferent seinerseits zeitnah an ihn weitergeleitete Titel bzw. mit Eintreffen der Datenlieferung einen Kauf entscheidet. Durch die Möglichkeit, zusätzlich individuelle Begriffe in den Titellisten zu markieren ${ }^{10}$, werden beispielsweise Verlage, auf die die WLB verzichtet, bereits an dieser Stelle aus dem Erwerbungsprozess genommen.

Mit Anawülasis können zudem weitere Geschäftsgänge digitalisiert werden: Die bislang wichtige Rückgabe von Papierkopien zu Titelhinweisen, die von der Landesbibliographie ausgewählt waren, aber vom Fachreferenten nicht gekauft wurden, entfällt nun. Die abgelehnten Titel werden automatisiert in das Modul Landesbibliographie geleitet und dort zum Nachweis in der Landesbibliographie-Datenbank voll elektronisch weiterverarbeitet. Außerdem fallen die monatlichen Ausdrucke der DNB-Reihe $\mathrm{H}$ weg, da diese ebenfalls mit Anawülasis ausgewertet wird.

Mittels Baden-Württemberg-Index können nun auch elektronische Publikationen aus der DNBReihe $\mathrm{O}$ zur Verfügung gestellt werden. Die Eingrenzung auf Baden-Württembergica soll die nicht zu leistende, umfassende, intellektuelle Prüfung auf sinnvolle Titel ersetzen. Außerdem ergeben sich Möglichkeiten zur Verwaltung von angeforderten Titeln, wenn diese vom Fachreferenten nach einem festgelegten Zeitraum (noch) nicht gekauft wurden.

Zum anderen weist Anawülasis auf (Titel-)Stichworte hin, die mit Orts- oder Personennamen der Lan- desbibliographie übereinstimmen. Trotz der Homonyme erbringen diese Vorschläge mit den Tipps den größten Teil der herauszufilternden Literatur. Zum dritten erlaubt die wegen der jetzt automatisiert gelieferten Tipps und Vorschläge gewonnene erhebliche Zeitersparnis die Überprüfung behandelter oder beteiligter Personen (Festschriften, Künstlerkataloge) über die angebotenen Links. Auf Papier erforderte das, konsequent gemacht, einen kaum vertretbaren Aufwand. Schließlich zeigen die sofort einzusehenden Inhaltsverzeichnisse von Sammelwerken auch unselbständige Titel von Relevanz, die im Papierzeitalter allzu oft erst durch Rezensionen oder andere Bibliographien gefunden wurden.

\section{Die Arbeit mit dem Modul Fachreferenten Was liefert Anawülasis?}

Anawülasis beliefert jeden Fachreferenten nur mit den Titeln seines Fachgebiets.. Es liefert außerdem direkt Titelhinweise von der Landesbibliographie und von anderen Fachreferenten oder Rückmeldungen von der MB Kauf. Für die Darstellung der Titellisten sind verschiedene Filter möglich (Abb. 8): Wochenlieferung, DNB-Reihe, DDC-Sachgruppe und WLB-Fachgruppe. Außerdem können verschiedene Datenquellen über das Auswertesystem bearbeitet werden. Das sind derzeit Titel der Deutschen Nationalbibliografie und der Buchhandlung Dreier sowie manuell dublizierte oder eingegebene Titel.

\section{Wie wird mit Anawülasis gearbeitet?}

1. Für jeden einzelnen Titel kann mit der Aktion „Kaufen“ (Icon: Warenkorb) und der Auswahl der WLB-Fachgruppe eine sofortige Kauf-Entscheidung getroffen oder mit "Erle-

\section{Stimme aus der "Landesbibliographie"}

Anawülasis bietet der Landesbibliographie bei der Ermittlung relevanter Literatur mehrfachen Nutzen. Zum einen gleicht das Programm die Personen-Schlagworte der DNB-Titel mit denen der Landesbibliographie ab und gibt Titel mit Übereinstimmung als Tipps heraus, so dass auch „entlegene Landeskinder" die dem Bearbeiter nicht unbedingt bewusst sind, zuverlässig erfasst werden. digen" (Icon Papiertonne) ein Kauf abgelehnt werden (Abb. 8). Erwerbungsentscheidungen können aber auch für mehrere Titel gleichzeitig über Massenfunktionen ( „alle Titel löschen") ausgelöst werden. Ist ein Titel mehreren Fachreferenten zugeordnet, kann jeder individuell eine der Aktionen 


\section{An\{3wälasıs}

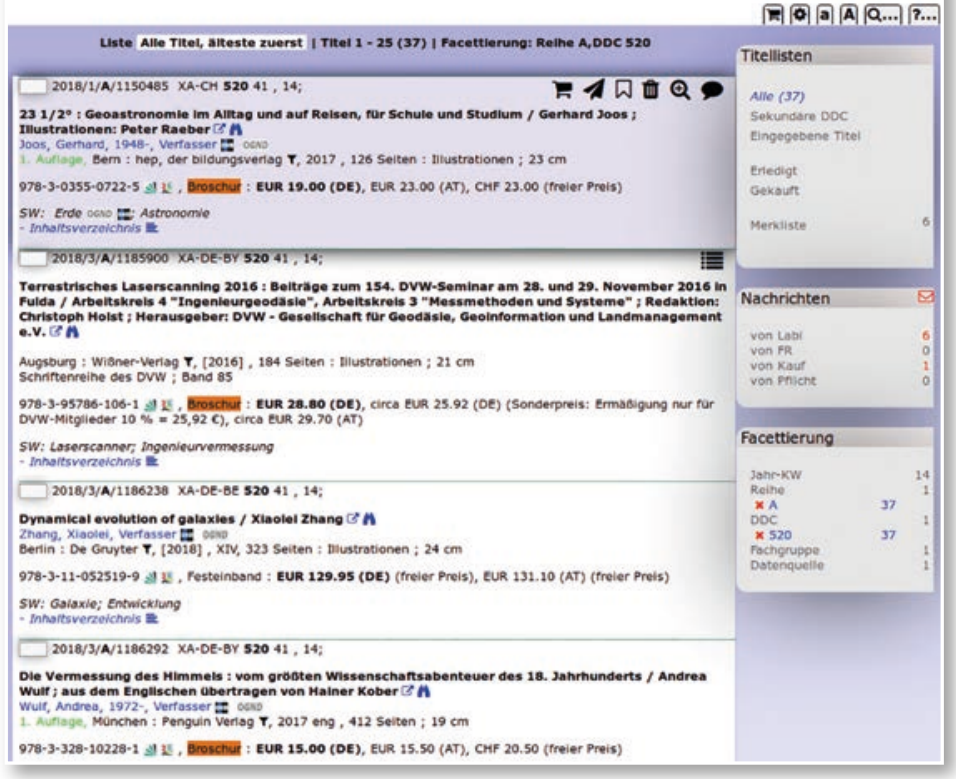

Abb. 8: Arbeitsoberfläche im Modul Fachreferent. Titelliste facettiert mit DNB-Reihe A, DDC-Sachgruppe 520

Löschen, Weiterleiten oder Merken durchführen, ohne dass dies Auswirkungen auf die Anzeige des Titels bei den übrigen Fachreferenten hat. Lediglich die Aktion „Kaufen" führt sofort zur Weiterleitung des Titels in das Modul Kauf und gleichzeitig zur Löschung des Titels bei allen anderen Fachreferenten.

Neu an Anawülasis ist, dass alle Fachreferenten einen landeskundlichen Titel für den Geschäftsgang Landesbibliographie direkt auswählen und somit Synergien geschaffen werden können. Mit der Auswahl „Für Labi" (Abb. 9) wird der Status des Titels auf "relevant für Labi" gesetzt und der Titel gleichzeitig aus dem Modul Landesbibliographie entfernt. Das erspart die Bearbeitung dieses Titels in der Landesbibliographie.

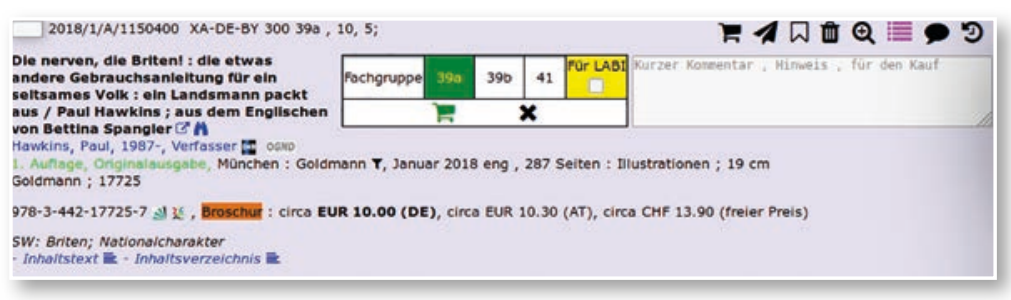

Abb. 9: Ausgewählter Titel mit Kriterien zur Kaufentscheidung

Individuell angelegte Merklisten (Icon Notizzettel) dienen der Verwaltung von Titeln, zum Beispiel für Rückstellungen mit späterer Kaufentscheidung oder für einen persönlichen Warenkorb. Ansonsten ist Anawülasis so konzipiert, dass eine Kaufent- scheidung sofort zur automatischen Weiteranleitung des Titels in die MB Kauf führt.

2. Soll ein anderer Fachreferent manuell über einen Titel informiert werden, kann dieser mit einem Kommentar, unter Auswahl des Fachreferentenkürzels direkt weitergeleitet werden (Icon Papierflieger „Weiterleiten an", Abb. 8). Dieser erscheint dann im Nachrichtenbereich des anderen Fachreferenten. Titel können ebenso an die Abteilung Landesbibliographie oder die MB Pflicht weitergeleitet werden.

3. Im Bereich "Nachrichten“ werden von der Landesbibliographie ausgewählte Titel, weitergeleitete Titel anderer Fachreferenten, der MB Kauf oder Pflicht angezeigt. Für Titel aus dem Nachrichtenbereich muss erneut eine Erwerbungsentscheidung oder Weiterleitung wie unter 1 bzw. 2 durchgeführt werden.

\section{Welche Vorteile bietet Anawülasis den Fachre- ferenten?}

Erhielten die Fachreferenten mit dem alten PDFGeschäftsgang entsprechend ihrem Fächerprofil mehr oder weniger umfangreiche Papier-Ausdrucke, so sind badische und württembergische Pflichttitel aus den Titellisten in Anawülasis entfernt. Je nach persönlicher Einstellung können zu bearbeitende Titellisten weiter reduziert werden, indem

- Grundaufnahmen mehrbändiger Werke ausgeblendet werden,

- Titel im Bestand der WLB ausgeblendet werden. ${ }^{11}$

- Übersetzungen oder Schulbücher automatisch erledigt werden können ${ }^{12}$,

- nach Jahr-Kalenderwoche, DNB-Reihe, DDC, WLB-Fachgruppe und Datenquelle facettiert wird. Die Titelanzeige bietet ihrerseits eine Auswahl an sinnvollen, schnell zugänglichen Zusatzinformationen wie Links zu Inhaltsverzeichnissen, Inhaltstexten und Normdatensätzen von Geographika und Personen, die zur GND der DNB und zur OnlineGND des Bibliotheksservicezentrums Baden-Württemberg führen. Über einen halbautomatischen Bestandsabgleich (über Titel, Verfasser) können Parallelausgaben ${ }^{13}$ oder gleichlautende Vorauflagen im SWB-Verbundkatalog abgerufen werden. Außerdem können individuelle Begriffe in der Titelanzeige markiert werden, zum Beispiel, wenn

11) Der Bestandsabgleich erkennt neben neuen auch retrospektive Titel im System.

12) Übersetzungen deutschsprachiger Werke, die im Ausland erschienen sind und Schulbücher werden i.d.R. nicht gekauft.

13) Zum Beispiel Paperback, Hardcover, elektronische Ressource. 
man auf Publikationen eines bestimmten Verlages aufmerksam gemacht werden möchte.

Erwerbungsentscheidungen können in Anawülasis nicht nur für digitale Bestellquellen getroffen werden, sondern auch für Titel aus gedruckten Verlagsprospekten, die über ein Blankoformular digitalisiert und in den elektronischen Geschäftsgang geleitet werden können. Bei einer Kaufentscheidung wird jeder Titel mit dem Namenskürzel des Fachreferenten und der voreingestellten ${ }^{14}$ oder frei ausgewählten WLB-Fachgruppe mit einem Klick (Abb. 9) an die MB Kauf weitergeleitet.

Mit der Papier-Auswertung waren die Fachreferenten von den vorangeschalteten Arbeitsgängen der Abteilung MB Pflicht und der Abteilung Landesbibliographie abhängig und mussten ggf. einige Tage bis Wochen auf die DNB-Titel warten. Einen der größten Mehrwerte bietet der Einsatz von Anawülasis durch den zeitlich unabhängigen Arbeitsbeginn der verschiedenen AuswerteStationen für die DNB-Titel. Außerdem entfällt eine "Bearbeitungs-Schleife", die die Fachreferenten weiter entlastet: Bei Titeln, die von der Landesbibliographie ausgewählt wurden, muss in der Folge des Geschäftsprozesses auch bei einer Kaufablehnung (Nichtkauf) nichts weiter beachtet werden. Musste sonst eine zusätzliche Papierkopie des Titels an die Landesbibliographie zurückgegeben werden, wird dieser automatisch vom System an die Landesbibliographie zurückgemeldet.

\section{Stimme aus dem „Fachreferat"}

Anawülasis hat meine Tätigkeit als Fachreferentin bei der Erwerbungsauswahl der Titel sehr erleichtert und verbessert. Für die Wirtschaftswissenschaften und allgemeinen Nachschlagewerke bin ich darauf angewiesen, dass aktuelle Neuerscheinungen möglichst schnell dem Benutzer zur Verfügung stehen. Durch die wöchentlichen Einspielungen der DNB- und Dreier-Titeldaten stehen die Erwerbungsunterlagen nun zeitnah zur Verfügung. Bei der Titelauswahl erleichtern die Verlinkungen zum SWB und KVK eine schnelle Überprüfung, ob der Titel bereits von der Bibliothek bestellt oder im Bestand vorhanden ist. Damit entfällt die bisherige manuelle Eingabe. Auch können ergänzend Preisangaben per Link zum OnlineBuchhandel ermittelt werden. Darüber hinaus ermöglichen verlinkte Inhaltsverzeichnisse eine verbesserte Entscheidung nach der Relevanz für den Bestandsaufbau des Fachgebiets. Per Mausklick lassen sich Titelvorschläge an andere Fachreferenten weiterleiten und entsprechend kommentieren. Wichtig sind die Merklisten, die nach eigenen Gruppierungen angelegt werden können. Damit können Titel zunächst zurückgestellt werden, bis eine Finanzierung aus dem Fachetat sichergestellt ist. Anawülasis ist vom Konzept her ein wichtiger Beitrag in Richtung papierloses Büro!
Wie wird mit Anawülasis gearbeitet?

1. In der Vollversion ist die Auswahl eines bestimmten Bestell-Titels möglich. Dafür muss dieser zur Bearbeitung reserviert werden (Icon "Schloss", Abb. 10), um zu verhindern, dass der Titel von einem weiteren Bearbeiter aufgerufen wird. Sollen beispielsweise sämtliche Bestellungen eines Fachreferenten oder einer WLBFachgruppe gesammelt aufgerufen werden, können über die entsprechende Facettierung auf diese Weise auch alle weiteren Titel gesperrt werden. Der eigentliche Bestellvorgang erfolgt nicht über Anawülasis, sondern über den Verbundkatalog (WinIBW) und das Lokalsystem aDIS/ BMS. Informationen wie die Relevanz für die Landesbibliographie oder Sonderstandorte (LesesaalSignaturen) werden in das Bestellkatalogisat übernommen. Anschließend 


\section{An\}\{3wülasıs}

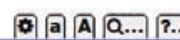

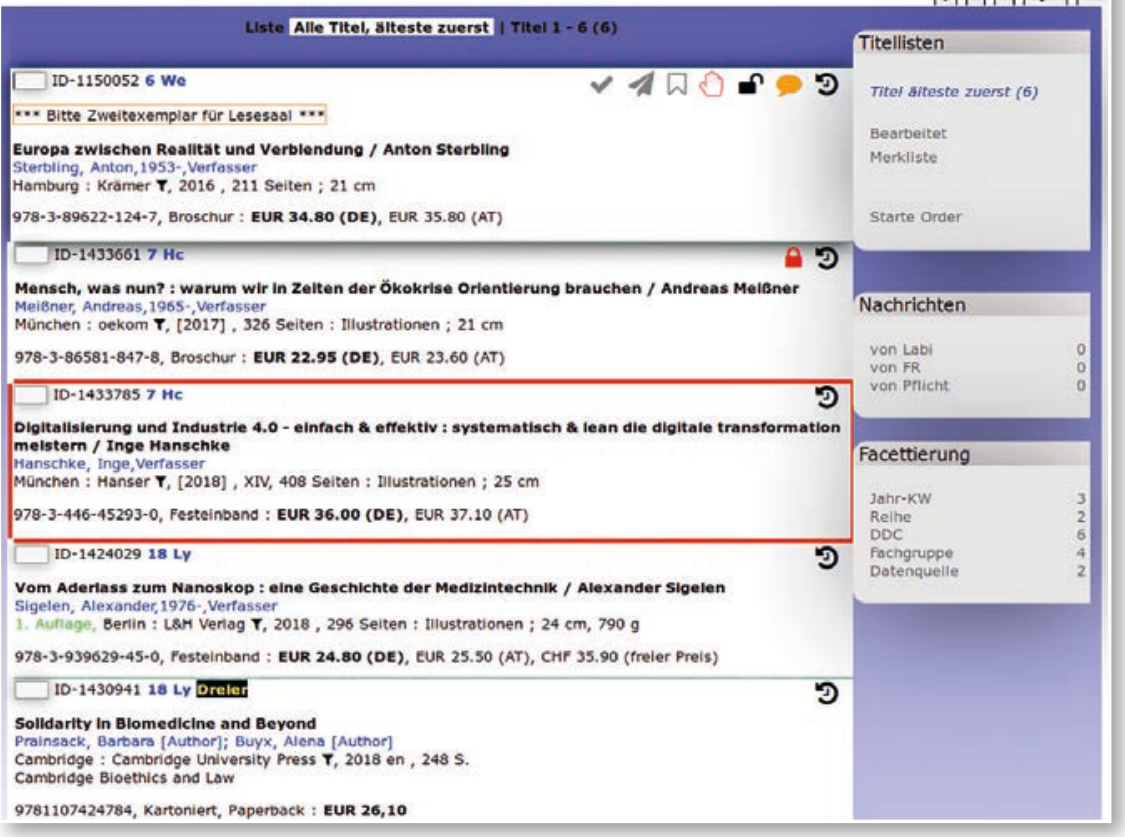

ne Titel automatisch für andere Bearbeiter gesperrt.

2. Rückmeldungen zu Titeln, die von anderen Modulen an die MB Kauf geschickt wurden, werden im "Nachrichten-Bereich" der Vollversion gelistet. Beispielsweise können Rückfragen zu vergriffenen Werken oder zur Bestellung einzelner Bände eines Gesamtwerkes direkt vom Fachreferenten beantwortet und an die MB Kauf geschickt werden.

\section{Welche Vorteile bietet Anawülasis der MB Kauf?}

Die MB Kauf muss mit zahlreichen Anwendungen gleichzeitig arbeiten. Sowohl Anawülasis als auch die Arbeitsumgebung wurden deshalb so

Abb. 10: Arbeitsoberfläche im Modul Kauf. Liste mit zu bestellenden Titeln, die für alle Bearbeiter aufrufbar sind und mit dem Icon "Schloss" zur Bearbeitung reserviert bzw. gesperrt werden können. Titel, die in diesem Moment von Kollegen bearbeitet werden, sind mit rotem Rahmen (Bearbeitung im One-Titel-Order-Fenster) bzw. rotem Schloss gekennzeichnet (Bearbeitung in Gesamtliste). konfiguriert, dass eine möglichst große Arbeitserleichterung erreicht wird:

- über die technische Ausstattung der Arbeitsplätze mit zwei Monitoren im Format 4:3 bzw. einem großen 24-Zoll-Bildschirm im Format 16:9,

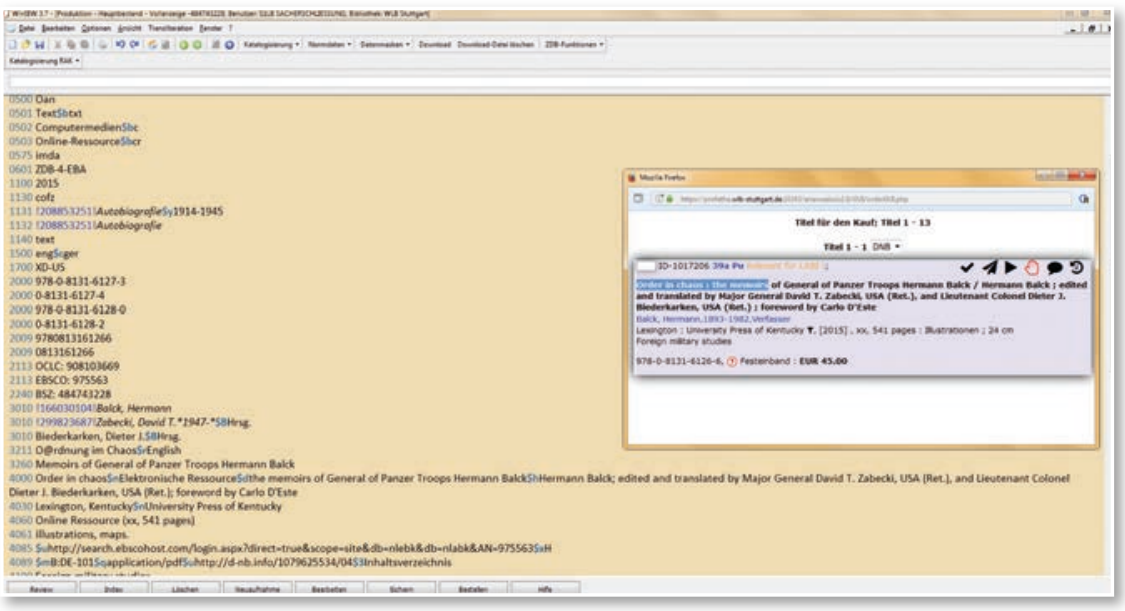

- durch die Bereitstellung von anwenderorientierten Versionen mit einer Vollversion und sämtlichen Funktionen oder mit einer übersichtlichen "1-Titelanzeige", in der ein Titel nach dem anderen eingeblendet wird,

- durch individuell einzustellende Titelanzeigen mit einer reduzierten Anzeige, die nur die notwendigsten bibliographischen Angaben und Bestellinformationen enthält, sowie einer Anzeige mit zusätzlichen Informationen und Links (z. B. zu Titelda-

Abb. 11: Zu bestellender Titel aus dem Order-Fenster, eingebettet in das Arbeitsfenster des SWB-Katalogs (WinIBW)

wechselt der Bearbeiter wieder zu Anawülasis und klickt auf das Icon „bestellt" (Icon „Haken“, Abb. 10). Durch diese Statusänderung wird der Titel aus dem Modul Kauf entfernt.

Die Reservierung eines Titels in Anawülasis (Icon "Schloss") kann jederzeit zurückgenommen werden; der Titel steht dann den anderen KaufMitarbeitern zur Verfügung.

Sämtliche Aktionen stehen auch in der One-TitelOrder-Version zur Verfügung, hier ist der aufgerufe- ten in anderen Katalogen oder zu Normdaten). Bestell-Titel aus verschiedenen Datenquellen können integriert über Anawülasis in die MB Kauf gelenkt und dort nach WLB-Fachgruppen sortiert werden, um beispielsweise Bestellungen für einen Lieferanten schnell, gesammelt und effizient zu bearbeiten. 
Mit der Einführung von Anawülasis hat sich in der MB 1 (in Anawülasis: Kauf) die wichtigste Bestellquelle grundlegend geändert. Unterschiedliche Funktionen wie beispielsweise das Nachrichtenfeld oder die Möglichkeit zur Weiterleitung von Titeln verbessern die Kommunikation mit anderen Abteilungen. Die Facettierung ermöglicht ein individuelles Arbeiten. An der Optimierung der Titellisten muss noch gearbeitet werden. Das Projektteam ist sehr aufgeschlossen, versucht alle Änderungsvorschläge umzusetzen und Fehler zeitnah zu korrigieren.

\section{Was leistet Anawülasis für die Württembergische Landesbibliothek?}

Mit Anawülasis wurde ein automatisierter und effizienter Geschäftsgang zur Auswertung von Titeldaten eingeführt. Allein von den $151.000 \mathrm{im}$ Jahr 2018 in den DNB-Reihen A und B verzeichneten Titeln, konnten 7.000 direkt der badischen und 15.500 Titel direkt der württembergischen Pflicht zugeordnet werden. Bei anderen Neuerscheinungsdiensten, die über keine Pflichterkennung verfügen, müssen Fachreferenten und Landesbibliographie sämtliche Titel sichten und erkennen, welche der Pflichtablieferung unterliegen - dank Anawülasis entfällt diese Aufgabe.

Das Auswertesystem läuft seit dem Produktivbetrieb im Oktober 2017 stabil und erfüllt nahezu alle Anwenderanforderungen. Als Inhouse-Entwicklung ist es vor allem auf landesbibliothekarische Aufgaben und Geschäftsgänge der WLB abgestimmt. Gegenüber sonstigen verfügbaren Systemen bietet Anawülasis damit eine Reihe von Vorteilen, weil die Anwendung:

- Titeldaten lieferantenunabhängig in unterschiedlichen Formaten über eine gemeinsame Oberfläche verarbeitet,
- Pflichttitel weitestgehend automatisiert erkennt und - nach einem Bestandsabgleich - nur zu reklamierende württembergische Titel ausliefert,

- die intellektuelle Auswahl von landeskundlicher Literatur zum Nachweis in der Landesbibliographie Baden-Württemberg berücksichtigt,

- den zeitlich parallelen Arbeitsbeginn aller Abteilungen (statt der linear abhängigen Bearbeitung durch MB Pflicht, Landesbibliographie und Fachreferenten) ermöglicht und zu einer enormen Beschleunigung von Auswertungs- und weiterer Erwerbungs-Workflows geführt hat. Von diesen Vorteilen können insbesondere Landesbibliotheken profitieren. Als erste Partnerin wird die Badische Landesbibliothek Anawülasis als Auswertesystem einsetzen. Beide Landesbibliotheken haben einen Überlassungsvertrag unterzeichnet, der die Einrichtung und Anpassung des Systems sowie ein Schulungskonzept regelt. Anawülasis ist in Karlsruhe mittlerweile eingerichtet und die Produktivsetzung für das Frühjahr 2019 geplant.

Jörg Oberfell / Anja Thalhofer 